\title{
Author Correction: Defining the relative and combined contribution of CTCF and CTCFL to genomic regulation
}

\author{
Mayilaadumveettil Nishana ${ }^{1}$, Caryn $\mathrm{Ha}^{1}$, Javier Rodriguez-Hernaez ${ }^{1}$, Ali Ranjbaran ${ }^{1}$, Erica Chio ${ }^{1}$, Elphege P. Nora ${ }^{2,3,4}$,
} Sana B. Badri ${ }^{1}$, Andreas Kloetgen ${ }^{5}$, Benoit G. Bruneau ${ }^{2,3,4,6}$, Aristotelis Tsirigos ${ }^{1,5}$ and Jane A. Skok ${ }^{1,7^{*}}$

The original article can be found online at https://doi.org/10.1186/ s13059-020-02024-0

* Correspondence: Jane.Skok@ nyumc.org; Jane.Skok@nyulangone. org

'Department of Pathology, New York University Langone Health, New York, NY 10016, USA ${ }^{7}$ Laura and Isaac Perlmutter Cancer Center, NYU School of Medicine, New York, NY 10016, USA

Full list of author information is available at the end of the article

\section{Correction to: Genome Biol 21, 108 (2020)}

https://doi.org/10.1186/s13059-020-02024-0

Following publication of the original paper [1], the authors reported an error in the acknowledgements section. The updated acknowledgements section is given below and the changes have been highlighted in bold typeface.

\section{Acknowledgements}

The authors thank Skok lab members for helpful scientific discussions, New York University School of Medicine High Performance Computing Facility (HPCF) for computing technical support, Adriana Heguy and the Genome Technology Center (GTC) core for sequencing efforts, Applied Bioinformatics Laboratories (ABL) for providing bioinformatics support and helping with the analysis and interpretation of the data and the NYU Flow Cytometry and Cell Sorting Center for FACS analysis and sorting. GTC and ABL are shared resources partially supported by the Cancer Center Support Grant P30CA016087 at the Laura and Isaac Perlmutter Cancer Center. The authors would like to acknowledge that Elphege Nora and Benoit Bruneau contributed unpublished cell lines for use in this article.

\section{Author details}

'Department of Pathology, New York University Langone Health, New York, NY 10016, USA. ${ }^{2}$ Gladstone Institutes, San Francisco, CA 94158, USA. ${ }^{3}$ Roddenberry Center for Stem Cell Biology and Medicine at Gladstone, San Francisco, CA 94158, USA. ${ }^{4}$ Cardiovascular Research Institute, University of California, San Francisco, CA 94158, USA. ${ }^{5}$ Applied Bioinformatics Laboratories, NYU School of Medicine, New York, NY 10016, USA. 'Department of Pediatrics, University of California, San Francisco, CA 94158, USA. 'Laura and Isaac Perlmutter Cancer Center, NYU School of Medicine, New York, NY 10016, USA

Published online: 02 June 2020

\section{Reference}

1. Nishana M, Ha C, Rodriguez-Hernaez J, et al. Defining the relative and combined contribution of CTCF and CTCFL to genomic regulation. Genome Biol. 2020;21:108 https://doi.org/10.1186/s13059-020-02024-0. 